\title{
Evolutionary Optimization of Yagi-Uda Antennas
}

\author{
Jibion D. Loha', William F. Krand', Derek S. Linden², and \\ Silvano P. Colombano' \\ - Computational Sciences Division. NASA Ames Research Center. \\ Wail Stop 269-1. Moffett Field, CA 94035-1000, USA \\ email: (jlohn, bkraus, colomban\}idemail arc nasa.gov \\ 2 Linclen Innovation Research, P.O Box 1601, Ashburn, VA, 20146. USA \\ email: dlinden 9 lindenir.com
}

\begin{abstract}
Yagi-Uda antennas are known to be difficult to design and optimize due to their sensitivity at bigh gain, and the inclusion of aumerous parasitic elements. We present a genetic algorithm-based automated antenna optimization system that uses a fixed Yagi-Uda topology and a byte-encoded antenns representation. The fitness calculation allows the implicit relationship between power gain and sidelobe/backlobe loss to emerge naturally, a technique that is less complex than previous approaches. The genetic operators used are also simpler. Our result$s$ include Yagi-Uda antennas that have excellent bandwidth and gain properties with very good impedance characteristics. Results exceeded previous Yagi-Uda antennas produced via evolutionary algorithms by at least $7.8 \%$ in mainlobe gain. We also present encouraging preliminary results where a coevolutionary genetic algorithm is used.
\end{abstract}

\section{Introduction}

Automated antenna synthesis via evolutionary design has recently garnered much attention in the research literature [12]. Underlying this enthusiasm is an issue that many designers readily acknowledge - good antenna design requires not only knowledge and intelligence, but experience and artistry. Thus automated design techniques and tools have been lacking. Evolutionary algorithms show promise because, among search algorithms, they are able to effectively search large, unknown design spaces.

The particular antenna we study in this paper is the Yagi-Uda, first proposed in 1926 [14]. We chose this type of antenna because it presents difficult design and optimization challenges, and because it was previously studied with respect to.evolutionary design [7]. The Yagi-Uda antenna is comprised of a set of parallel elements with one reflector element, one driven element (driven from its center), and one or more director elements (see Fig. 1). The highest gain can be achieved along the axis and on the side with the directors. The reflector element reflects power forwards and thus acts like a small ground plane. The design parameters consist of element lengths, inter-element spacings, and element diameters. 


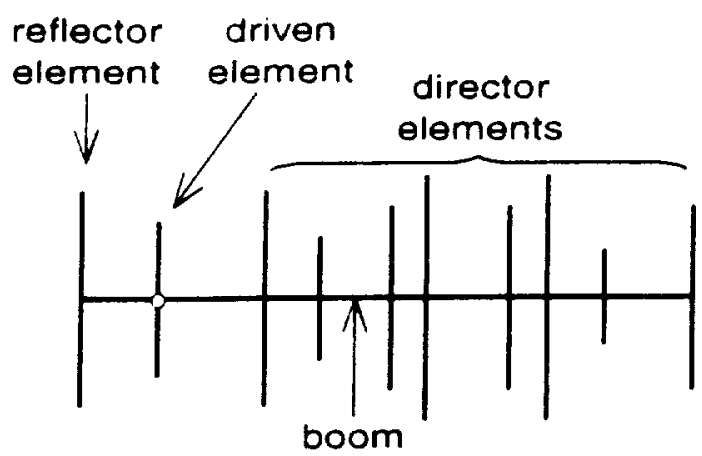

Fig. 1. Typical Yagi-Uda antenna.

The application that we use is taken from [7]. It involves designing a special feed for the Arecibo 305-meter spherical reflector in Puerto Rico [3]. The antenna was to be used to search for primeval hydrogen having a redshift of approximately 5. Neutral hydrogen line emission is at a frequency of $1420 \mathrm{MHz}$; thus the frequency region of interest was about $235 \mathrm{MHz}$. Preliminary studies indicated that the band from 219 to $251 \mathrm{MHz}$ was of the greatest interest, particularly from 223 to $243 \mathrm{MHz}$. The most important design goal was for the feed to have sidelobes/backlobes at least $25 \mathrm{~dB}$ down from the mainbeam gain in the region from $70^{\circ}<\phi<290^{\circ}$, due to the interference which came from surrounding radio and TV towers. Of lesser importance was that the E-plane (the plane parallel to the plane of the antenna) and H-plane (perpendicular to the E-plane) beamwidths be about $50^{\circ}$.

Voltage Standing Wave Ratio, or VSWR, is a way to quantify reflectedwave interference, and thus the amount of impedance mismatch at the junction. VSWR is the ratio between the highest voltage and the lowest voltage in the signal envelope along a transmission line [13]. The VSWR was desired to be less than 3 and the gain was to be maximized, limited by the wide beamwidth. The feed would be mounted over a 1.17 meter square ground plane-that is, a ground plane only $0.92 \lambda$ in size.

\section{Antenna Representation and Operators}

The representational scheme used is similar to that taken from [7]. As shown in Fig. 2, this scheme is comprised of 14 elements, each one encoding a length and spacing value. Each floating point value was encoded as three bytes, yielding a resolution of $1 / 2^{24}$ per value. The first pair of values encoded the reflector element, the second pair encoded the driven element, and the remaining 12 pairs encoded the directors. One point crossover was used with cut points allowed between bytes. Mutation was applied on individual bytes. 

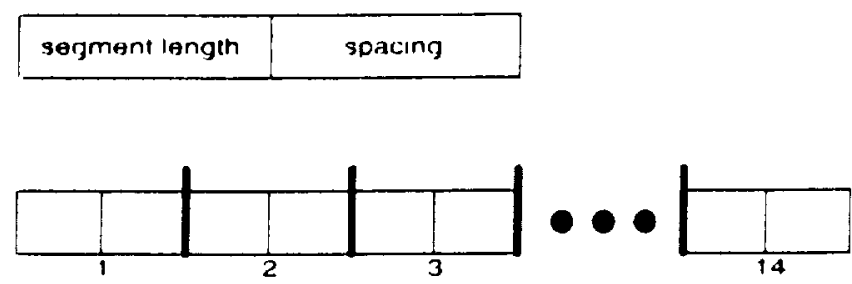

Fig. 2. Genetic representation of a 14-element Yagi-Uda antenna.

Radius values were constrained to $2,3,4,5$, or $6 \mathrm{~mm}$. All elements within a given individual were assigned the same radius value. Element lengths were constrained to be symmetric around the $x$-axis and between 0 and $1.5 \lambda$. Elements having zero length were removed from the antenna; as a consequence, a constructed antenna could have less than 14 elements. Spacing between adjacent elements (along the $z$ axis) was constrained to be between $0.05 \lambda$ and $0.75 \lambda$. The wavelength $\lambda$ was 1.195 meters, the wavelength of $235 \mathrm{MHz}$.

\section{Experimental Setup}

Experiments were set up as follows. The NEC2 simulation program [4] was used to evaluate all antenna designs. We used a parallel master/slave generational genetic algorithm with a population size of 6000 . One point crossover across byte boundaries was used at a rate of $80 \%$. Mutation was uniform across bytes at a rate of $1 \%$. Runs were executed on a 32-node Beowulf computing cluster [11].

The wire geometry encoded by each individual chromosome was first translated into a NEC input deck, which was subsequently sent to the NEC2 simulator. The segment size for all elements was fixed at $0.1 \lambda$, where $\lambda$ was the wavelength corresponding to $235 \mathrm{MHz}$. The source element for excitation was specified to be the middle segment of the driven element. The $z$ location of the reflector element was always set to 0 . The antenna was analyzed in free space.

The simulator was instructed to sample the radiation pattern of each individual at three different frequency values: 219,235 , and $251 \mathrm{MHz}$, representing a $13.6 \%$ bandwidth. Each radiation pattern was calculated at $\phi$ set to $0^{\circ}$ and $\theta$ varying between $0^{\circ}$ and $355^{\circ}$, the latter sampled at $5^{\circ}$ increments. VSWR values were also calculated for each of the three frequencies.

Fitness was expressed as a cost function to be minimized. The calculation was as follows:

$$
\text { - } F=-G_{L}+\sum\left(C * V_{i}\right)
$$

where: $G_{L}=$ lowest gain of all frequencies measured at $\theta=0^{\circ}$ and $\phi=0^{\circ}, V_{i}=$ VSWR at the ith frequency, and

$$
C=\left\{\begin{array}{l}
0.1 \text { if } V_{1} \leq 3 \\
1 \text { if } V_{1}>3
\end{array}\right.
$$


Carking from this ralculation wis a term involving sidelobe/hatklohe attenua-

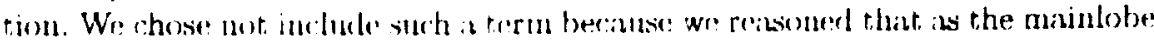

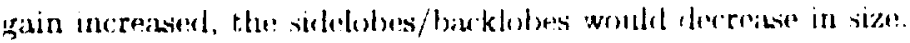

\section{Experimental Results}

Thirteen runs were executed under liffering random number streams for comparison purposes. Table $I$ summarizes the run clata for the best antenna found in each run of 100 generations. Fig. 3 shows the radiation pattern from the best antenna found (run 13). It exhibits $10.58 \mathrm{~dB}$ and has a VSWR of 2.02 at its center frequency. Its sidelobe/backlobe gain at this frequency is $3.07 \mathrm{~dB}$. Fig. 4 shows a diagram of the antenna's physical structure.

To increase simulation speed, the evolved antennas were produced without the presence of a ground plane - an idealized setting. Adding a ground plane thus simulates more realistic conditions. We removed the reflector element and simulated the best antennas found over a ground plane of 1.17 meters [7]. We found the performance increased - at the center frequency the mainlobe gain was $12.52 \mathrm{~dB}$ and the VSWR was 2.39. At $291 \mathrm{MHz}$, the gain was $11.33 \mathrm{~dB}$, and at $251 \mathrm{MHz}$, the gain was $11.15 \mathrm{~dB}$. In contrast, the antenna produced in [7] exhibits gains of $10.36,10.91,10.34 \mathrm{~dB}$ at 219,235 , and $251 \mathrm{~dB}$, respectively. Thus the antenna from run 13 has a minimum performance increase of $7.8 \%$ as compared to the previously reported antenna.

\begin{tabular}{|c|c|c|c|c|c|c|}
\hline \multirow[b]{2}{*}{ Run } & \multicolumn{2}{|c|}{$219 \mathrm{MHz}$} & \multicolumn{2}{|c|}{$235 \mathrm{MHz}$} & \multicolumn{2}{|c|}{$251 \mathrm{MHz}$} \\
\hline & $\mathrm{dB}$ & VSWR & $\mathrm{dB}$ & TSWR & $\mathrm{dB}$ & VSWR \\
\hline 1 & 9.63 & 2.33 & 9.64 & 1.67 & 10.20 & 2.99 \\
\hline 2 & 9.49 & 2.23 & 9.08 & 1.85 & 9.20 & 1.58 \\
\hline 3 & 9.23 & 2.89 & 10.04 & 1.11 & 9.62 & 2.60 \\
\hline 4 & 9.24 & 2.47 & 9.23 & 1.35 & 9.37 & 2.83 \\
\hline 5 & 8.73 & 2.83 & 8.79 & 1.51 & 9.22 & 2.60 \\
\hline 6 & 9.35 & 2.87 & 9.51 & 1.73 & 9.28 & 2.00 \\
\hline 7 & 9.87 & 2.64 & 9.82 & 1.99 & 9.46 & 1.98 \\
\hline 8 & 9.04 & 2.35 & 9.02 & 1.64 & 9.08 & 2.92 \\
\hline 9 & 9.44 & 2.96 & $\overline{9.46}$ & 1.87 & 9.51 & 2.39 \\
\hline 10 & 9.02 & 1.25 & 9.12 & 2.42 & 9.02 & 1.41 \\
\hline 11 & 10.01 & 1.95 & 9.81 & 1.97 & 10.11 & 1.66 \\
\hline 12 & 9.37 & 2.55 & 9.17 & 1.70 & 9.41 & 2.47 \\
\hline 13 & 10.34 & 2.57 & 10.58 & 2.02 & 10.51 & 1.70 \\
\hline
\end{tabular}

Table 1. Results from the best individual after 100 generations for each of the 13 runs ( $\mathrm{dB}$ is measured at $\phi=0^{\circ}, \theta=0^{\circ}$ ). 

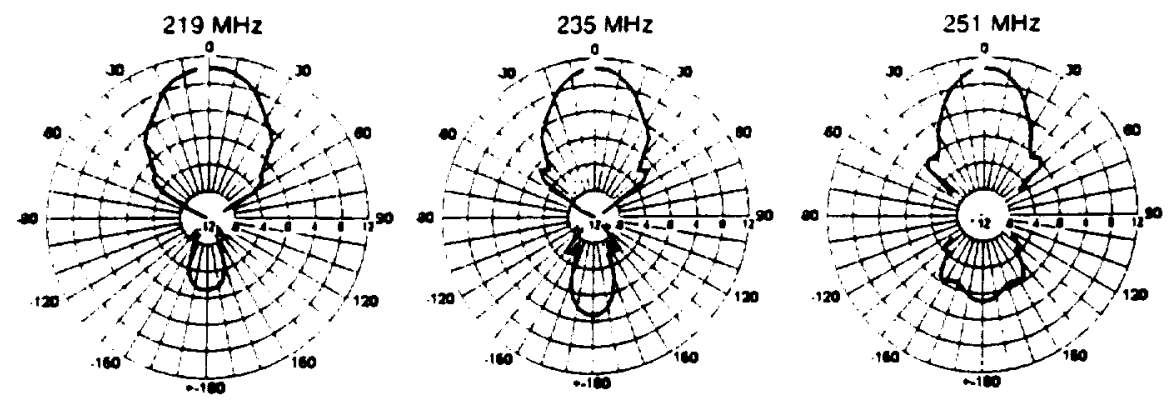

Fig. 3. Radiation pattern of the best evolved antenna without a ground plane, measured at $0^{\circ} \leq \theta<360^{\circ}, \phi=0^{\circ}$, for 219,235 , and $251 \mathrm{MHz}$, respectively. (The scale is $2 \mathrm{~dB}$ per division. Inner ring is $-12 \mathrm{db}$, outer ring is $12 \mathrm{~dB}$.)

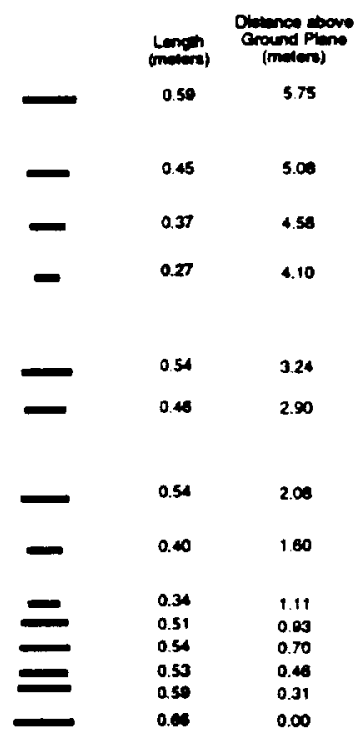

Fig. 4. The best Yagi-Uda antenna from run 13. The radius of all elements was $3 \mathrm{~mm}$. 


\section{Coevolutionary Algorithm - Method and Preliminary Results}

A coevolutionary genetic algerithm wis also applied to the intenna optimization problem describerl above. The experiments are ongoing is of this writing, and we briefly mention some encouraging initial results. The algorithun used is similar to that presenter in $[9 \mid$. Two populations are used: one consisting of antenna designs as described ibove. and one consisting of target vectors. The fundamental idea is that the target vectors encapsulate level-of-difficulty. Then, under the control of the genetic algorithm, the target vectors evolve from easy to difficult based on the level of proficiency of the antenna population.

Each target vector consists of a set of objectives that must be met in order for a target vector to be "solved." A target vector consisting of two values: the mainlobe gain (in $d B$ ) and a VSWR value. A target vector was considered to be solved by a given antenna if:

$$
G_{\text {target }}<G_{L} \quad \text { and } \quad V_{\text {target }}>V_{L}
$$

where $G_{L}$ is lowest gain of all frequencies measured at $\theta=0^{\circ}$ and $\phi=0^{\circ}$, and $V_{L}$ is lowest VSWR of all frequencies. For example, an antenna with a $G_{L}$ value equal to $5 \mathrm{~dB}$ and a $V_{L}$ value equal to 8 would solve the target vector $\langle 2,12\rangle$ but not $\langle 7,12\rangle$.

Values for target gain ranged between $0 \mathrm{~dB}$ (easy) and $12 \mathrm{~dB}$ (difficult). Target VSWR values ranged between 12 (easy) and 3 (difficult). Target vectors are represented as a list of floating point values that are mutated individually by randomly adding or subtracting a small amount (5\% of the largest legal value). Single point crossover was used, and crossover points were chosen between the values.

The general form of the fitness calculations are from [9]. In summary, antennas are rewarded for solving difficult target vectors. The most difficult target vector is defined to be the target vector that only one antenna can solve. Such a target vector garners the highest fitness score. Target vectors that are unsolvable, or are very easy to solve by the current antenna population, are given low fitness scores.

We ran our coevolutionary algorithm for 200 generations using 1600 individuals in both populations. In the antenna population, crossover and mutation rates were 0.8 and 0.1 , respectively. In the target vector population, crossover and mutation rates were $0.8,0.5$, respectively.

The highest-fitness individual came from generation 199. It had mainlobe gains of $8.30,8.51$, and $8.30 \mathrm{~dB}$ at 219,235 , and $251 \mathrm{MHz}$, respectively. While performance is less than the runs from above, it was achieved with a much smaller population, and it is currently our single data point.

Fig. 5 shows a plot of how the highest fitness target vectors varied during the run. Such plots can give insight regarding the difficulty of achieving one objective at the expense of another. In the plot, we see that difficult VSWR levels (near 3.0) are attainable early on and remain so throughout the run. The algorithm 


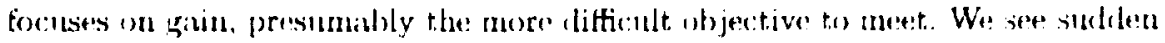
jumps in gatu near gentrattions 13 and 190 , accompanted by rolibations in the VSWR.

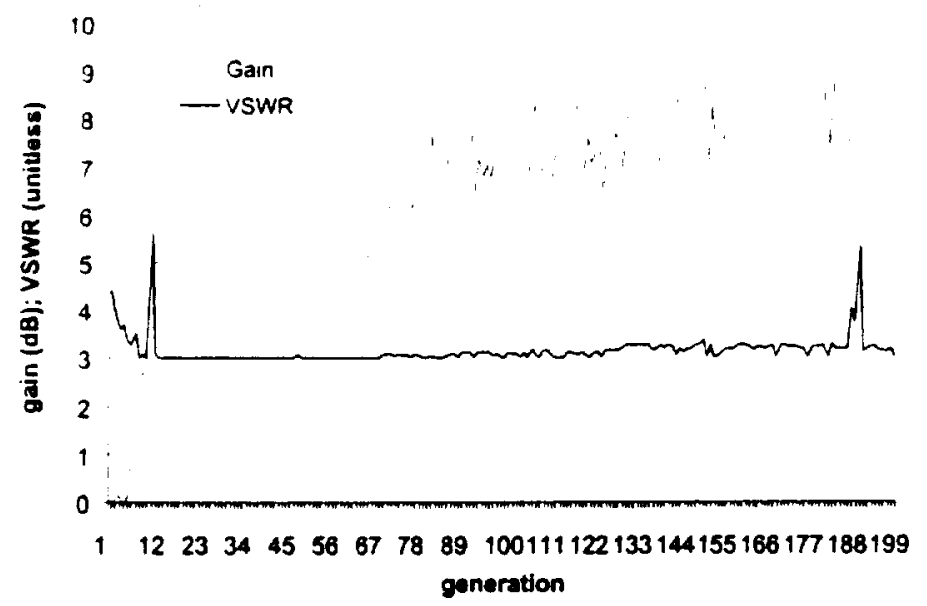

Fig. 5. Coevolution run: plot of gain and VSWR for the best target vector over 200 generations.

\section{Discussion}

Small improvements in antenna performance can be significant in many applications. Because of their numerous design variables, complex behavior, and sensitivity to parameters, Yagi-Uda antennas are notoriousily difficult to optimize. Our experiments produced several excellent antennas in a relatively small number of generations. When simulated over a finite ground plane, the highest performance antenna found exhibiting a mainlobe gain that was $7.8 \%$ higher than a previously-reported antenna.

Previous work has explicitly included a sidelobe/backlobe term in the fitness function in order to minimize radiation outside of the desired direction [7]. We did not include an explicit sidelobe/backlobe term but rather relied on the fact that the radiation pattern of an antenna is a zero sum quantity - increasing the intensity in one direction will implicitly reduce the amount of radiation in other directions.

Finally, we are encouraged by our preliminary results produced using coevolutionary optimization. There we saw an antenna generated that had very good properties while requiring less evaluations than the standard GA approach. 


\section{Acknowledgments}

The: research described in this papen wis performed at . VASA Antes Research Center. and wist sponsored by the NASA Intelligent Systems Program. Rarliation pattern plots were male using NEC-Win Plus software from Nittiny Scientific.

\section{References}

1. E.E. Altshuler and D.S. Linden. "Design of a Loaded Monopole Having Hemispherical Coverage Using a Genetic Algorithm." IEEE Trans. on Antennas and Propagation. Vol. 45, No. 1, January 1997.

2. E.E. Altshuler and D.S. Linden. "Wire Antenna Designs using a Genetic Algorithm." IEEE Antenna \& Propagation Society Mag., Vol. 39, pp. 33-43, April 1997.

3. I.M. Avruch, et al. "A Spectroscopic Search for Protoclusters at High Redshift." Bulletin of the American Astron. Society, Vol. 27, No. 4, 1995.

4. G.J. Burke and A.J. Poggio. "Numerical Electromagnetics Code (NEC)-Method of moments." Rep. UCID18834, Lawrence Livermore Laboratory, Jan. 1981.

5. C.D. Chapman, K. Saitou, M.J. Jakiela. "Genetic Algorithms as an Approach to Configuration and Topology Design." J. Mechanical Des., Vol. 116, December 1994.

6. J.H. Holland, Adaptation in Natural and Artificial Systems, Univ. of Michigan Press, Ann Arbor, 1975.

7. D.S. Linden, "Automated Design and Optimization of Wire Antennas using Genetic Algorithms." Ph.D. Thesis, MIT, September 1997.

8. D.S. Linden and E.E. Altsbuler. "Automating Wire Antenna Design using Genetic Algorithms." Microwave Journal, Vol. 39, No. 3, March 1996.

9. J.D. Lohn, G.L. Haith, S.P. Colombano, D. Stassinopoulos, "A Comparison of Dynamic Fitness Schedules for Evolutionary Design of Amplifiers," Proc. of the First NASA/DoD Workshop on Evolvable Hardware, Pasadena, CA, IEEE Computer Society Press, 1999, pp. 87-92.

10. J.D. Lohn, S.P. Colombano, "A Circuit Representation Technique for Automated Circuit Design," IEEE Transactions on Evolutionary Computation, vol. 3, no. 3, 1999, pp. 205-219.

11. J.D. Lohn, S.P. Colombano, G.L. Haith, D. Stassinopoulos, "A Parallel Genetic Algorithm for Automated Electronic Circuit Design," Proc. of the Computational Aerosciences Workshop, NASA Ames Research Center, Feb. 2000.

12. Electromagnetic Optimization by Genetic Algorithms. Y. Rahmat-Samii and E. Michielssen, eds., Wiley, 1999.

13. D. H. Staelin, et al. 6.014: Electromagnetic Waves (course notes). MIT, May, 1992.

14. S. Uda and Y. Mushiake, Yagi-Uda Antenna, Maruzden, Tokyo, 1954. 